JOURNAL OF OPTIMIZATION, DIFFERENTIAL EQUATIONS AND THEIR APPLICATIONS (JODEA) Volume 26, Issue 2, December 2018, pp. 55-67, DOI 10.15421/141810

ISSN (print) 2617-0108

ISSN (on-line) $\mathrm{xxxx}-\mathrm{xxxx}$

\title{
A FORMULATION OF AN EVOLUTION EQUATION GOVERNING MAGNETIC LINES
}

Vladimir L. Borsch*

\section{Communicated by Prof. V. Ye. Belozyorov}

\begin{abstract}
It is shown that 'a free function' in the evolution equation of Hornig \& Schindler for the magnetic induction (Physics of Plasmas, 3 (3), 781-791) has a unique representation, obtained in an explicit form. Some conclusions of the explicit formulation of the evolution equation are discussed.
\end{abstract}

Key words: magnetic induction, magnetic lines.

2010 Mathematics Subject Classification: 49J20, 35J20, 35B45, 35B65.

\section{Introduction to the formulation}

The magnetic induction equation

$$
\boldsymbol{B}_{t}+\nabla \times(\boldsymbol{B} \times \boldsymbol{u})=\mathbf{0}
$$

is a constitutive part of the governing equations of ideal MHD [5]. In (1.1) $\boldsymbol{u}(t, \boldsymbol{x})$ is the velocity field of a moving continuum, $\boldsymbol{B}(t, \boldsymbol{x})$ is the magnetic induction field, $(t, \boldsymbol{x})$ refers to an inertial frame of reference, and the lower index $t$ indicates the partial derivative with respect to $t$.

The magnetic induction equation has been studying by many authors, but in the current study our concern is the following equation

$$
\boldsymbol{B}_{t}+\boldsymbol{w} \cdot \nabla \boldsymbol{B}-\boldsymbol{B} \cdot \nabla \boldsymbol{w}=\lambda \boldsymbol{B},
$$

derived by Hornig \& Schindler [7] for the evolution of the $\boldsymbol{B}$-field and discussed in $[2,3,11]$. In $(1.2) \boldsymbol{w}(t, \boldsymbol{x})$ is the velocity of the magnetic lines (the vector lines of the $\boldsymbol{B}$-field), $\lambda$ is 'a scalar free function'.

If the solenoidal nature of the $\boldsymbol{B}$-field $(\nabla \cdot \boldsymbol{B}=0)$ is accounted for in (1.1), then the former converts into the equation

$$
\boldsymbol{B}_{t}+\boldsymbol{u} \cdot \nabla \boldsymbol{B}-\boldsymbol{B} \cdot \nabla \boldsymbol{u}+(\nabla \cdot \boldsymbol{u}) \boldsymbol{B}=\mathbf{0},
$$

exactly the same as the Zorawski criterion [13] for the $\boldsymbol{B}$-field to be frozen in the moving continuum. It follows from (1.3) that $\boldsymbol{w}_{\perp}=\boldsymbol{u}_{\perp}$ and generally $\boldsymbol{w}_{\|} \neq \boldsymbol{u}_{\|}$, where $\boldsymbol{w}(t, \boldsymbol{x})=\boldsymbol{w}_{\perp}(t, \boldsymbol{x})+\boldsymbol{w}_{\|}(t, \boldsymbol{x}), \boldsymbol{u}(t, \boldsymbol{x})=\boldsymbol{u}_{\perp}(t, \boldsymbol{x})+\boldsymbol{u}_{\|}(t, \boldsymbol{x})$, and the lower

\footnotetext{
*Dept. of Differential Equations, Faculty of Mech \& Math, Oles Honchar Dnipro National University, 72, Gagarin av., Dnipro, 49010, Ukraine, bvl@dsu.dp.ua

(C) Vladimir L. Borsch, 2018.
} 
indices mean respectively local orthogonal and tangent directions to a magnetic line. But (1.2) is surely to differ from the criterion [13], and from this there stems our interest to the formulation (1.2) of the evolution equation for $\boldsymbol{B}(t, \boldsymbol{x})$ and especially to function $\lambda$.

\section{Preliminaries of the formulation}

Following [7] we introduce some diffeomorphic mappings to study the formulation (1.2) of the evolution equation for the magnetic induction.

The first one

$$
\boldsymbol{x}=\boldsymbol{\varphi}_{\boldsymbol{u}}(t, \boldsymbol{X}), \quad \boldsymbol{X} \in \mathcal{D}\left(t^{\prime}\right) \subseteq \mathbf{R}^{3}, \quad t \geqslant t^{\prime},
$$

maps domain $\mathcal{D}\left(t^{\prime}\right)$ onto domain $\mathcal{D}(t), t \geqslant t^{\prime}$, where $\boldsymbol{X}$ are coordinates parametrizing domain $\mathcal{D}\left(t^{\prime}\right)$ (sometimes called the Lagrangian independent variables), and

$$
\boldsymbol{X}=\varphi_{\boldsymbol{u}}\left(t^{\prime}, \boldsymbol{X}\right)
$$

is the identical mapping. The inverse mapping

$$
\boldsymbol{X}=\boldsymbol{\psi}_{\boldsymbol{u}}(t, \boldsymbol{x}), \quad \boldsymbol{x} \in \mathcal{D}(t), \quad t \geqslant t^{\prime},
$$

acts in the opposite direction (in time).

The partial derivative of (2.1) with respect to time is called the velocity of the mapping

$$
\boldsymbol{v}(t, \boldsymbol{X})=\frac{\partial \boldsymbol{\varphi}_{\boldsymbol{u}}(t, \boldsymbol{X})}{\partial t}
$$

and is easily presented in the Eulerian independent variables $\boldsymbol{x}$

$$
\boldsymbol{u}(t, \boldsymbol{x})=\boldsymbol{v}\left(t, \boldsymbol{\psi}_{\boldsymbol{u}}(t, \boldsymbol{x})\right) .
$$

Diffeomorphism (2.1) is responsible for the motion of the continuum and is the only solution to the following Cauchy problem 'in the whole'

$$
\left\{\begin{array}{l}
\frac{\mathrm{d} \boldsymbol{x}}{\mathrm{d} t}=\boldsymbol{u}(t, \boldsymbol{x}), \\
\boldsymbol{x}\left(t^{\prime}\right)=\boldsymbol{X}^{\prime} .
\end{array}\right.
$$

The second one

$$
\boldsymbol{x}=\boldsymbol{\varphi}_{\boldsymbol{w}}(t, \boldsymbol{X}), \quad \boldsymbol{X} \in \mathcal{D}\left(t^{\prime}\right) \subseteq \mathbf{R}^{3}, \quad t \geqslant t^{\prime},
$$

maps domain $\mathcal{D}\left(t^{\prime}\right)$ onto domain $\mathcal{D}(t), t \geqslant t^{\prime}$, and

$$
\boldsymbol{X}=\varphi_{\boldsymbol{w}}\left(t^{\prime}, \boldsymbol{X}\right)
$$

is the identical mapping. The inverse mapping 


$$
\boldsymbol{X}=\boldsymbol{\psi}_{\boldsymbol{w}}(t, \boldsymbol{x}), \quad \boldsymbol{x} \in \mathcal{D}(t), \quad t \geqslant t^{\prime},
$$

acts in the opposite direction (in time).

The partial derivative of (2.7) with respect to time is called the velocity of the mapping

$$
\boldsymbol{w}(t, \boldsymbol{x})=\left.\frac{\partial \boldsymbol{\varphi}_{\boldsymbol{w}}(t, \boldsymbol{X})}{\partial t}\right|_{\boldsymbol{X}=\boldsymbol{\psi}_{\boldsymbol{w}}(t, \boldsymbol{x})} .
$$

Diffeomorphism (2.7) is responsible for the motion (evolution) of magnetic field $\boldsymbol{B}(t, \boldsymbol{x})$ and can be specified through velocity field $\boldsymbol{w}(t, \boldsymbol{x})$. Newcomb [9] and Stern [12] discussed some ways of specifying velocity filed $\boldsymbol{w}(t, \boldsymbol{x})$ having properties mentioned above.

The solutions to the following Cauchy problem

$$
\left\{\begin{array}{l}
\frac{\mathrm{d} \boldsymbol{x}}{\mathrm{d} \sigma}=\boldsymbol{B}(t, \boldsymbol{x}), \\
\boldsymbol{x}\left(\sigma^{\prime}\right)=\boldsymbol{x}^{\prime},
\end{array} \quad \boldsymbol{x}, \boldsymbol{x}^{\prime} \in \mathcal{D}(t), \quad t \geqslant t^{\prime},\right.
$$

are called the magnetic lines (of the $\boldsymbol{B}$ field) and are given as the following diffeomorphism (the third one)

$$
\boldsymbol{x}=\varphi_{\boldsymbol{B}}\left(t, \boldsymbol{x}^{\prime}, \sigma\right),
$$

where $\sigma$ is a scalar parameter along the magnetic lines.

Diffeomorphism (2.12) is the only solution to the Cauchy problem (2.11), since

$$
\boldsymbol{B}(t, \boldsymbol{x})=\left.\frac{\partial \boldsymbol{\varphi}_{\boldsymbol{B}}\left(t, \boldsymbol{x}^{\prime}, \sigma\right)}{\partial \sigma}\right|_{\left(\boldsymbol{x}^{\prime}, \sigma\right) \stackrel{(2.12)}{\rightarrow} \boldsymbol{x}} .
$$

We note that $\varphi_{\boldsymbol{u}}, \boldsymbol{\varphi}_{\boldsymbol{w}}$ and $\varphi_{\boldsymbol{B}}$ are called sometimes the (phase) flows for fields $\boldsymbol{u}(t, \boldsymbol{x}), \boldsymbol{w}(t, \boldsymbol{x})$ and $\boldsymbol{B}(t, \boldsymbol{x})$, whereas in [3] $\boldsymbol{\varphi}_{\boldsymbol{w}}$ and $\boldsymbol{\varphi}_{\boldsymbol{B}}$ are called the generating functions for $\boldsymbol{w}(t, \boldsymbol{x})$ and $\boldsymbol{B}(t, \boldsymbol{x})$.

\section{The main proposition of the formulation}

Now we present a fully geometrical proof of the formulation (1.2) of the evolution equation for the magnetic induction extended compared to that given in [7]. The proof is based on a reparametrization of diffeomorphism $\varphi_{B}$ introduced in [7] and the commutation condition of flows (proposition 4.2.27 in [1]).

Proposition 3.1. If diffeomorphisms $\boldsymbol{\varphi}_{\boldsymbol{w}}(2.7)$ and $\boldsymbol{\varphi}_{\boldsymbol{B}}$ (2.12) commute, the latter being reparametrized the proper way; then the resulted evolution equation for the $\boldsymbol{B}$-field is determined uniquely. 
Proof. Let an arbitrary instant $t^{\prime}$ be the reference one. This means that the Cartesian coordinates $\boldsymbol{x}$ at $t^{\prime}$ are considered to be the Lagrangian ones: $\boldsymbol{X}=\boldsymbol{x}$. Then take a magnetic line $\Gamma\left(t^{\prime}\right)$ and parametrize it due to diffeomorphism $\varphi_{B}$ as follows

$$
\boldsymbol{x}=\varphi_{\boldsymbol{B}}\left(t^{\prime}, \boldsymbol{x}_{\mathrm{M}}, \sigma\right),
$$

where $\mathbf{M} \in \Gamma\left(t^{\prime}\right)$ is an arbitrary point: $\boldsymbol{x}_{\mathbf{M}}=\boldsymbol{\varphi}_{\boldsymbol{B}}\left(t^{\prime}, \boldsymbol{x}_{\mathbf{M}}, \sigma_{\mathbf{M}}\right)$. Choosing an infinitesimal increment $\Delta \sigma$ of the parameter we determine point $\mathbf{N} \in \Gamma\left(t^{\prime}\right)$ :

$$
\boldsymbol{x}_{\mathbf{N}}=\boldsymbol{\varphi}_{\boldsymbol{B}}\left(t^{\prime}, \boldsymbol{x}_{\mathbf{M}}, \sigma_{\mathbf{N}}\right), \quad \sigma_{\mathbf{N}}=\sigma_{\mathbf{M}}+\Delta \sigma .
$$

At instant $t^{\prime}+\Delta t$, where $\Delta t$ is an infinitesimal increment of time, diffeomorphism $\varphi_{w}$ maps magnetic line $\Gamma\left(t^{\prime}\right)$ onto a magnetic line $\Gamma\left(t^{\prime}+\Delta t\right)$. The latter is the image of the former due to diffeomorphism $\varphi_{u}$, if we assume that the magnetic lines are 'frozen' in the moving continuum, this implies that $\boldsymbol{w}_{\perp}=\boldsymbol{u}_{\perp}$ and generally $\boldsymbol{w}_{\|} \neq \boldsymbol{u}_{\|}$.

Diffeomorphism $\boldsymbol{\varphi}_{\boldsymbol{w}}$ maps points $\mathbf{M}, \mathbf{N} \in \Gamma\left(t^{\prime}\right)$ into points $\mathbf{M}^{\prime}, \mathbf{N}^{\prime} \in \Gamma\left(t^{\prime}+\Delta t\right)$ as follows

$$
\left\{\begin{array}{l}
x_{\mathbf{M}^{\prime}}=\varphi_{\boldsymbol{w}}\left(t^{\prime}+\Delta t, X_{\mathbf{M}}\right), \\
x_{\mathbf{N}^{\prime}}=\varphi_{\boldsymbol{w}}\left(t^{\prime}+\Delta t, X_{\mathbf{N}}\right),
\end{array}\right.
$$

and finally, at instant $t^{\prime}+\Delta t$ diffeomorphism $\varphi_{B}$ maps point $\mathbf{M}^{\prime}$ into point $\mathbf{N}^{\prime \prime}$

$$
\boldsymbol{x}_{\mathbf{N}^{\prime \prime}}=\varphi_{\boldsymbol{B}}\left(t^{\prime}+\Delta t, \boldsymbol{x}_{\mathbf{M}^{\prime}}, \sigma_{\mathbf{M}^{\prime \prime}}\right),
$$

where $\sigma_{\mathbf{M}^{\prime \prime}}=\sigma_{\mathbf{M}^{\prime}}+\Delta \sigma$, and $\boldsymbol{x}_{\mathbf{M}^{\prime}}=\boldsymbol{\varphi}_{\boldsymbol{B}}\left(t^{\prime}+\Delta t, \boldsymbol{x}_{\mathbf{M}^{\prime}}, \sigma_{\mathbf{M}^{\prime}}\right)$.

Generally speaking, $\mathbf{N}^{\prime \prime} \neq \mathbf{N}^{\prime}$, that is there is no commutation of diffeomorphisms $\varphi_{\boldsymbol{w}}$ and $\boldsymbol{\varphi}_{\boldsymbol{B}}$ (Fig. 1,a).

It is clear that for commutation to occur, the parametrization of magnetic lines due to diffeomorphism $\varphi_{B}$ should be changed as

$$
\boldsymbol{x}=\overline{\boldsymbol{\varphi}}_{\boldsymbol{B}}\left(t, \boldsymbol{x}^{\prime}, \alpha\left(t, \boldsymbol{x}^{\prime}, \sigma\right)\right), \quad t \geqslant t^{\prime},
$$

where $\alpha\left(t^{\prime}, \boldsymbol{x}^{\prime}, \sigma\right)=\sigma$, and the bar over the symbol of the diffeomorphism denotes the proper reparametrization. The evident restriction to be imposed on function $\alpha$ along magnetic line $\Gamma\left(t^{\prime}+\Delta t\right)$ for diffeomorphisms $\varphi_{w}, \varphi_{B}$ and $\bar{\varphi}_{B}$ to commute (Fig. 1,b) is the following

$$
\boldsymbol{x}_{\mathbf{N}^{\prime}}=\bar{\varphi}_{\boldsymbol{B}}\left(t^{\prime}+\Delta t, \boldsymbol{x}_{\mathbf{M}^{\prime}}, \alpha_{\mathbf{N}^{\prime}}\right),
$$

where

$$
\alpha_{\mathbf{N}^{\prime}}=\sigma_{\mathbf{M}^{\prime}}+\Delta \alpha, \quad \Delta \alpha=\left.\frac{\partial \alpha\left(t^{\prime}+\Delta t, \boldsymbol{x}_{\mathbf{M}^{\prime}}, \sigma\right)}{\partial \sigma}\right|_{\sigma=\sigma_{\mathbf{M}^{\prime}}} \Delta \sigma .
$$



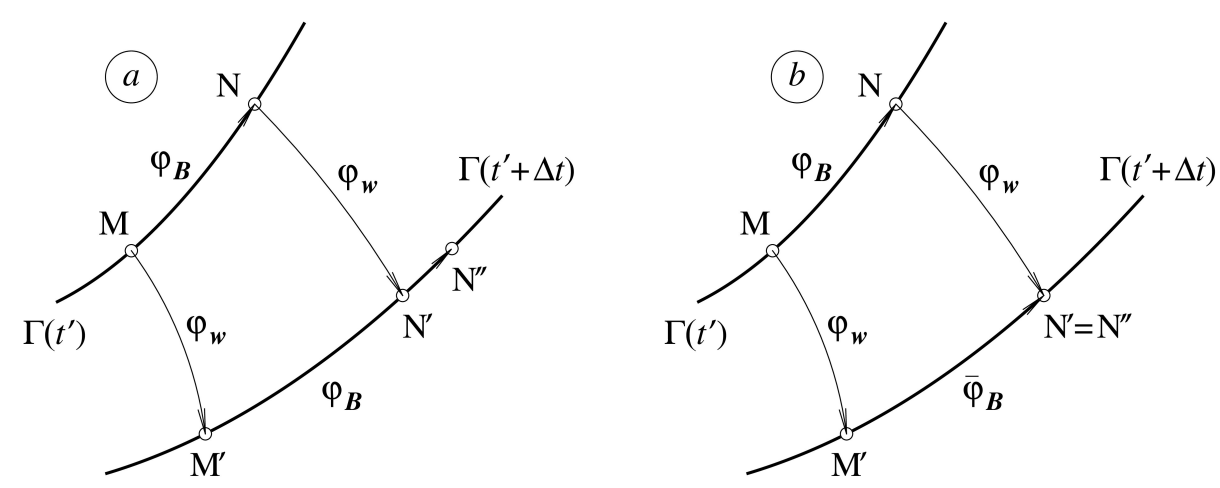

Fig. 1. Evolution of magnetic line $\Gamma$ between two instants $t^{\prime}$ and $t^{\prime}+\Delta t$ : infinitesimal quadrilaterals $\mathbf{M M}^{\prime} \mathbf{N}^{\prime} \mathbf{N}$ formed by diffeomorphisms $\varphi_{w}$ and $\varphi_{B}$ without commutation $\left(a: \mathbf{N}^{\prime} \neq \mathbf{N}^{\prime \prime}\right)$ and by diffeomorphisms $\varphi_{\boldsymbol{w}}, \boldsymbol{\varphi}_{B}$ and $\bar{\varphi}_{B}$ with commutation $\left(b: \mathbf{N}^{\prime}=\mathbf{N}^{\prime \prime}\right)$

But we can easily find $\Delta \alpha$ directly, without finding function $\alpha$. Indeed, for the reparametrized diffeomorphism $\bar{\varphi}_{\boldsymbol{B}}$ we have (one should refer to (2.11) and replace $\sigma$ with $\alpha$ in the differential equation for the magnetic lines)

$$
\frac{\mathrm{d} \boldsymbol{x}}{\mathrm{d} \alpha}=\boldsymbol{B}
$$

hence, the required expression for $\Delta \alpha$ in $(3.2),(3.3)$ is

$$
\Delta \alpha=\frac{\left|\boldsymbol{x}_{\mathbf{N}^{\prime}}-\boldsymbol{x}_{\mathbf{M}^{\prime}}\right|}{\left|\boldsymbol{B}\left(t^{\prime}+\Delta t, \boldsymbol{x}_{\mathbf{M}^{\prime}}\right)\right|} .
$$

Expanding the expressions for the coordinates of points $\mathbf{N}, \mathbf{M}^{\prime}$ and $\mathbf{N}^{\prime}$ into series with respect to $\Delta t$ and $\Delta \sigma$ and retaining only the terms of the first order in $\Delta t$ and $\Delta \sigma$ we obtain

$$
\begin{gathered}
\boldsymbol{x}_{\mathbf{N}}=\boldsymbol{\varphi}_{\boldsymbol{B}}\left(t^{\prime}, \boldsymbol{x}_{\mathbf{M}}, \sigma_{\mathbf{M}}\right)+\Delta \sigma \frac{\partial \boldsymbol{\varphi}_{\boldsymbol{B}}\left(t^{\prime}, \boldsymbol{x}_{\mathbf{M}}, \sigma_{\mathbf{N}}\right)}{\partial \sigma}=\boldsymbol{x}_{\mathbf{M}}+\Delta \sigma \boldsymbol{B}\left(t^{\prime}, \boldsymbol{x}_{\mathbf{M}}\right), \\
\left\{\begin{array}{l}
\boldsymbol{x}_{\mathbf{M}^{\prime}}=\boldsymbol{\varphi}_{\boldsymbol{w}}\left(t^{\prime}, \boldsymbol{X}_{\mathbf{M}}\right)+\Delta t \frac{\partial \boldsymbol{\varphi}_{\boldsymbol{w}}\left(t^{\prime}, \boldsymbol{X}_{\mathbf{M}}\right)}{\partial t} \stackrel{(2.8)}{=} \boldsymbol{x}_{\mathbf{M}}+\Delta t \boldsymbol{w}\left(t^{\prime}, \boldsymbol{x}_{\mathbf{M}}\right), \\
\boldsymbol{x}_{\mathbf{N}^{\prime}}=\boldsymbol{\varphi}_{\boldsymbol{w}}\left(t^{\prime}, \boldsymbol{X}_{\mathbf{N}}\right)+\Delta t \frac{\partial \boldsymbol{\varphi}_{\boldsymbol{w}}\left(t^{\prime}, \boldsymbol{X}_{\mathbf{N}}\right)}{\partial t} \stackrel{(2.8)}{=} \boldsymbol{x}_{\mathbf{N}}+\Delta t \boldsymbol{w}\left(t^{\prime}, \boldsymbol{x}_{\mathbf{N}}\right)
\end{array}\right.
\end{gathered}
$$

Using the obtained expressions for the difference $\boldsymbol{x}_{\mathbf{N}^{\prime}}-\boldsymbol{x}_{\mathrm{M}^{\prime}}$ yields to 


$$
\left\{\begin{aligned}
\boldsymbol{x}_{\mathbf{N}^{\prime}}-\boldsymbol{x}_{\mathbf{M}^{\prime}} & \stackrel{(3.5)}{=} \boldsymbol{x}_{\mathbf{N}}-\boldsymbol{x}_{\mathbf{M}}+\Delta t\left(\boldsymbol{w}\left(t^{\prime}, \boldsymbol{x}_{\mathbf{N}}\right)-\boldsymbol{w}\left(t^{\prime}, \boldsymbol{x}_{\mathbf{M}}\right)\right) \\
\stackrel{(3.4)}{=} & \Delta \sigma \boldsymbol{B}\left(t^{\prime}, \boldsymbol{x}_{\mathbf{M}}\right)+\Delta t\left(\boldsymbol{w}\left(t^{\prime}, \boldsymbol{x}_{\mathbf{M}}+\Delta \sigma \boldsymbol{B}\left(t^{\prime}, \boldsymbol{x}_{\mathbf{M}}\right)\right)-\boldsymbol{w}\left(t^{\prime}, \boldsymbol{x}_{\mathbf{M}}\right)\right) \\
& =\Delta \sigma \boldsymbol{B}\left(t^{\prime}, \boldsymbol{x}_{\mathbf{M}}\right)+\Delta t \Delta \sigma \boldsymbol{B}\left(t^{\prime}, \boldsymbol{x}_{\mathbf{M}}\right) \cdot \nabla \boldsymbol{w}\left(t^{\prime}, \boldsymbol{x}_{\mathbf{M}}\right),
\end{aligned}\right.
$$

and the required expression for the proper value of $\Delta \alpha$ reduces to the following

$$
\Delta \alpha=\frac{\left|\boldsymbol{B}\left(t^{\prime}, \boldsymbol{x}_{\mathbf{M}}\right)+\Delta t \boldsymbol{B}\left(t^{\prime}, \boldsymbol{x}_{\mathbf{M}}\right) \cdot \nabla \boldsymbol{w}\left(t^{\prime}, \boldsymbol{x}_{\mathbf{M}}\right)\right|}{\left|\boldsymbol{B}\left(t, \boldsymbol{x}_{\mathbf{M}}\right)+\Delta t \frac{\partial \boldsymbol{B}\left(t^{\prime}, \boldsymbol{x}_{\mathbf{M}}\right)}{\partial t}+\Delta t \boldsymbol{w}\left(t^{\prime}, \boldsymbol{x}_{\mathbf{M}}\right) \cdot \nabla \boldsymbol{B}\left(t^{\prime}, \boldsymbol{x}_{\mathbf{M}}\right)\right|} \Delta \sigma
$$

Now we consider infinitesimal quadrilateral $\mathbf{M M}^{\prime} \mathbf{N}^{\prime} \mathbf{N}$ (Fig. 1,b) and set up the following commutation equality for diffeomorphisms $\varphi_{w}, \varphi_{B}$ and $\bar{\varphi}_{B}$ acting on $\mathbf{M M}^{\prime} \mathbf{N}^{\prime} \mathbf{N}$

$$
\overline{\boldsymbol{\varphi}}_{\boldsymbol{B}}\left(t^{\prime}+\Delta t, \boldsymbol{\varphi}_{\boldsymbol{w}}\left(t^{\prime}+\Delta t, \boldsymbol{X}_{\mathbf{M}}\right), \sigma_{\mathbf{N}^{\prime}}\right)=\boldsymbol{\varphi}_{\boldsymbol{w}}\left(t^{\prime}+\Delta t, \boldsymbol{\varphi}_{\boldsymbol{B}}\left(t^{\prime}, \boldsymbol{x}_{\mathbf{M}}, \sigma_{\mathbf{N}}\right)\right) .
$$

The necessary and sufficient condition for the above equality to hold is the following commutation condition [1]

$$
\left[\frac{\partial^{2}}{\partial \sigma \partial t} \overline{\boldsymbol{\varphi}}_{\boldsymbol{B}}\left(t, \boldsymbol{\varphi}_{\boldsymbol{w}}(t, \boldsymbol{X}), \alpha(t, \boldsymbol{x}, \sigma)\right)\right]=\left[\frac{\partial^{2}}{\partial t \partial \sigma} \boldsymbol{\varphi}_{\boldsymbol{w}}\left(t, \boldsymbol{\varphi}_{\boldsymbol{B}}(t, \boldsymbol{x}, \sigma)\right)\right],
$$

where the brackets herein after denote that a quantity enclosed is calculated at point $\left(t^{\prime}, \boldsymbol{x}_{\mathbf{M}}\right)$. Using the defined derivatives (2.10) and (2.13) of $\boldsymbol{\varphi}_{\boldsymbol{w}}$ and $\boldsymbol{\varphi}_{\boldsymbol{B}}$ in (3.8) yields to

$$
\frac{\partial}{\partial t}\left[\boldsymbol{B}\left(t, \boldsymbol{\varphi}_{\boldsymbol{w}}(t, \boldsymbol{X})\right) \frac{\partial}{\partial \sigma} \alpha(t, \boldsymbol{x}, \sigma)\right]=\frac{\partial}{\partial \sigma}\left[\boldsymbol{w}\left(t, \boldsymbol{\varphi}_{\boldsymbol{B}}(t, \boldsymbol{x}, \sigma)\right)\right] .
$$

The derivative of the term in the brackets at the right hand side of the above equality is evident

$$
\frac{\partial \boldsymbol{\varphi}_{\boldsymbol{B}}\left(t^{\prime}, \boldsymbol{x}_{\mathbf{M}}, \sigma_{\mathbf{M}}\right)}{\partial \sigma} \cdot \nabla \boldsymbol{w}\left(t^{\prime}, \boldsymbol{x}_{\mathbf{M}}\right) \equiv \boldsymbol{B}\left(t^{\prime}, \boldsymbol{x}_{\mathbf{M}}\right) \cdot \nabla \boldsymbol{w}\left(t^{\prime}, \boldsymbol{x}_{\mathbf{M}}\right),
$$

and the same is true for the product in the brackets at the left hand side

$$
\begin{aligned}
& \left(\frac{\partial \boldsymbol{B}\left(t^{\prime}, \boldsymbol{x}_{\mathbf{M}}\right)}{\partial t}+\frac{\partial \boldsymbol{\varphi}_{\boldsymbol{w}}\left(t^{\prime}, \boldsymbol{x}_{\mathbf{M}}\right)}{\partial t} \cdot \nabla \boldsymbol{B}\left(t^{\prime}, \boldsymbol{x}_{\mathbf{M}}\right)\right)\left[\frac{\partial \alpha}{\partial \sigma}\right]+\boldsymbol{B}\left(t^{\prime}, \boldsymbol{x}_{\mathbf{M}}\right)\left[\frac{\partial^{2} \alpha}{\partial \sigma \partial t}\right] \\
\equiv & \left(\frac{\partial \boldsymbol{B}\left(t^{\prime}, \boldsymbol{x}_{\mathbf{M}}\right)}{\partial t}+\boldsymbol{w}\left(t^{\prime}, \boldsymbol{x}_{\mathbf{M}}\right) \cdot \nabla \boldsymbol{B}\left(t^{\prime}, \boldsymbol{x}_{\mathbf{M}}\right)\right)\left[\frac{\partial \alpha}{\partial \sigma}\right]+\boldsymbol{B}\left(t^{\prime}, \boldsymbol{x}_{\mathbf{M}}\right)\left[\frac{\partial^{2} \alpha}{\partial \sigma \partial t}\right] .
\end{aligned}
$$


To calculate the first derivative on $\sigma$ and the mixed derivative of function $\alpha$ we use the divided differences as follows

$$
\begin{aligned}
{\left[\frac{\partial \alpha}{\partial \sigma}\right] } & =\lim _{\substack{\Delta t \rightarrow 0 \\
\Delta \sigma \rightarrow 0}} \frac{\left|\boldsymbol{x}_{\mathbf{N}^{\prime}}-\boldsymbol{x}_{\mathbf{M}^{\prime}}\right|}{\left|\boldsymbol{B}\left(t^{\prime}+\Delta t, \boldsymbol{x}_{\mathbf{M}^{\prime}}\right)\right| \Delta \sigma}=\lim _{\substack{\Delta t \rightarrow 0 \\
\Delta \sigma \rightarrow 0}} \frac{\left|\boldsymbol{x}_{\mathbf{N}^{\prime}}-\boldsymbol{x}_{\mathbf{M}^{\prime}}\right|\left|\boldsymbol{B}\left(t^{\prime}, \boldsymbol{x}_{\mathbf{M}}\right)\right|}{\left|\boldsymbol{x}_{\mathbf{N}}-\boldsymbol{x}_{\mathbf{M}}\right|\left|\boldsymbol{B}\left(t^{\prime}+\Delta t, \boldsymbol{x}_{\mathbf{M}^{\prime}}\right)\right|}=1, \\
& =\lim _{\substack{\Delta t \rightarrow 0 \\
\Delta \sigma \rightarrow 0}} \frac{\left|\boldsymbol{x}_{\mathbf{N}^{\prime}}-\boldsymbol{x}_{\mathbf{M}^{\prime}}\right|\left|\boldsymbol{B}\left(t^{\prime}, \boldsymbol{x}_{\mathbf{M}}\right)\right|}{\left|\boldsymbol{x}_{\mathbf{N}}-\boldsymbol{x}_{\mathbf{M}}\right|\left|\boldsymbol{B}\left(t^{\prime}+\Delta t, \boldsymbol{x}_{\mathbf{M}^{\prime}}\right)\right|}=1, \\
{\left[\frac{\partial^{2} \alpha}{\partial t \partial \sigma}\right] } & =\lim _{\substack{\Delta t \rightarrow 0 \\
\Delta \sigma \rightarrow 0}} \frac{\frac{\left|\boldsymbol{x}_{\mathbf{N}^{\prime}}-\boldsymbol{x}_{\mathbf{M}^{\prime}}\right|}{\left|\boldsymbol{B}\left(t^{\prime}+\Delta t, \boldsymbol{x}_{\mathbf{M}^{\prime}}\right)\right|}-\frac{\left|\boldsymbol{x}_{\mathbf{N}}-\boldsymbol{x}_{\mathbf{M}}\right|}{\left|\boldsymbol{B}\left(t^{\prime}, \boldsymbol{x}_{\mathbf{M}}\right)\right|}}{\Delta \sigma \Delta t}=\lim _{\Delta t \rightarrow 0} \frac{\frac{\Delta \alpha}{\Delta \sigma}-1}{\Delta t} .
\end{aligned}
$$

For completing the calculation of the mixed derivative we consider the numerator of the last expression separately as follows

$$
\begin{aligned}
& \frac{\Delta \alpha}{\Delta \sigma}-1 \stackrel{(3.6)}{=} \frac{[|\boldsymbol{B}+\Delta t \boldsymbol{B} \cdot \nabla \boldsymbol{w}|]}{\left[\left|\boldsymbol{B}+\Delta t \frac{\partial \boldsymbol{B}}{\partial t}+\Delta t \boldsymbol{w} \cdot \nabla \boldsymbol{B}\right|\right]}-1 \\
= & \frac{\left[|\boldsymbol{B}+\Delta t \boldsymbol{B} \cdot \nabla \boldsymbol{w}|-\left|\boldsymbol{B}+\Delta t \frac{\partial \boldsymbol{B}}{\partial t}+\Delta t \boldsymbol{w} \cdot \nabla \boldsymbol{B}\right|\right]}{\left[\left|\boldsymbol{B}+\Delta t \frac{\partial \boldsymbol{B}}{\partial t}+\Delta t \boldsymbol{w} \cdot \nabla \boldsymbol{B}\right|\right]} \\
\times & \frac{\left[|\boldsymbol{B}+\Delta t \boldsymbol{B} \cdot \nabla \boldsymbol{w}|+\left|\boldsymbol{B}+\Delta t \frac{\partial \boldsymbol{B}}{\partial t}+\Delta t \boldsymbol{w} \cdot \nabla \boldsymbol{B}\right|\right]}{\left[|\boldsymbol{B}+\Delta t \boldsymbol{B} \cdot \nabla \boldsymbol{w}|+\left|\boldsymbol{B}+\Delta t \frac{\partial \boldsymbol{B}}{\partial t}+\Delta t \boldsymbol{w} \cdot \nabla \boldsymbol{B}\right|\right]} \\
= & \frac{\left[(\boldsymbol{B}+\Delta t \boldsymbol{B} \cdot \nabla \boldsymbol{w})^{2}-\left(\boldsymbol{B}+\Delta t \frac{\partial \boldsymbol{B}}{\partial t}+\Delta t \boldsymbol{w} \cdot \nabla \boldsymbol{B}\right)^{2}\right]}{\left[\left|\boldsymbol{B}+\Delta t \frac{\partial \boldsymbol{B}}{\partial t}+\Delta t \boldsymbol{w} \cdot \nabla \boldsymbol{B}\right|\right]\left[|\boldsymbol{B}+\Delta t \boldsymbol{B} \cdot \nabla \boldsymbol{w}|+\left|\boldsymbol{B}+\Delta t \frac{\partial \boldsymbol{B}}{\partial t}+\Delta t \boldsymbol{w} \cdot \nabla \boldsymbol{B}\right|\right]} \\
= & \frac{\left[\left|\boldsymbol{B}+\Delta t \frac{\partial \boldsymbol{B}}{\partial t}+\Delta t \boldsymbol{w} \cdot \nabla \boldsymbol{B}\right|\right]\left[|\boldsymbol{B}+\Delta t \boldsymbol{B} \cdot \nabla \boldsymbol{w}|+\left|\boldsymbol{B}+\Delta t \frac{\partial \boldsymbol{B}}{\partial t}+\Delta t \boldsymbol{w} \cdot \nabla \boldsymbol{B}\right|\right]}{\left[\boldsymbol{B} \cdot(\nabla \boldsymbol{w}) \cdot \boldsymbol{B}-\boldsymbol{B} \cdot \frac{\partial \boldsymbol{B}}{\partial t}-\boldsymbol{w} \cdot(\nabla \boldsymbol{B}) \cdot \boldsymbol{B}+\mathcal{O}(\Delta t)\right]}
\end{aligned}
$$


Replacing the numerator of the expression for the mixed derivative of function $\alpha$ with the above one and taking the limit at $\Delta t \rightarrow 0$ we obtain the final expression for the mixed derivative

$$
\left[\frac{\partial^{2} \alpha}{\partial t \partial \sigma}\right] \equiv-\lambda=-\left[\frac{1}{|\boldsymbol{B}|^{2}}\left(\frac{1}{2} \frac{\partial|\boldsymbol{B}|^{2}}{\partial t}+\boldsymbol{w} \cdot(\nabla \boldsymbol{B}) \cdot \boldsymbol{B}-\boldsymbol{B} \cdot(\nabla \boldsymbol{w}) \cdot \boldsymbol{B}\right)\right] .
$$

Gathering all the terms obtained when treating commutation condition (3.8) we conclude that the required evolution equation for the magnetic induction reads

$$
\boldsymbol{B}_{t}+\boldsymbol{w} \cdot \nabla \boldsymbol{B}-\boldsymbol{B} \cdot \nabla \boldsymbol{w}=\underbrace{\frac{1}{|\boldsymbol{B}|^{2}}\left(\frac{1}{2} \frac{\partial|\boldsymbol{B}|^{2}}{\partial t}+\boldsymbol{w} \cdot(\nabla \boldsymbol{B}) \cdot \boldsymbol{B}-\boldsymbol{B} \cdot(\nabla \boldsymbol{w}) \cdot \boldsymbol{B}\right)}_{\lambda} \boldsymbol{B} .
$$

Arbitrariness of point $\left(t^{\prime}, \boldsymbol{x}_{\mathbf{M}}\right)$ means that the equation obtained is valid at any point $(t, \boldsymbol{x}) \in \mathcal{D}(t)$ and this is denoted by dropping the brackets referring to point $\left(t^{\prime}, \boldsymbol{x}_{\mathbf{M}}\right)$ in $(3.8)$.

\section{The Galilean invariance of the formulation}

The MHD phenomena in the non-relativistic limit are Galilean invariant [10], but the original magnetic induction equation (1.1) does not obey the Galilean transformation. It was Godunov [6] who showed that (1.1) transforms to formulation (1.3) being Galilean invariant if the solenoidal nature of the $\boldsymbol{B}$-field is accounted for explicitly. And what about evolution equation (3.10)?

Proposition 4.1. Evolution equation (3.10) is Galilean invariant.

Proof. Let $(\tau, \boldsymbol{\xi})$ be an inertial frame of reference such that

$$
\left\{\begin{array}{l}
\tau=t \\
\boldsymbol{\xi}=\boldsymbol{x}-t \boldsymbol{a}
\end{array}\right.
$$

where $\boldsymbol{a}$ is a constant velocity, then velocity field $\boldsymbol{w}(t, \boldsymbol{x})$ and magnetic induction $\boldsymbol{B}(t, \boldsymbol{x})$ observed in $(\tau, \boldsymbol{\xi})$ and indicated by an asterisk are

$$
\left\{\begin{array}{l}
\boldsymbol{w}^{*}(\tau, \boldsymbol{\xi})=\boldsymbol{w}(t, \boldsymbol{x})-\boldsymbol{a} \\
\boldsymbol{B}^{*}(\tau, \boldsymbol{\xi})=\boldsymbol{B}(t, \boldsymbol{x})
\end{array}\right.
$$

When changing the frame of reference from $(t, \boldsymbol{x})$ to $(\tau, \boldsymbol{\xi})$ the partial derivatives transform as follows 


$$
\begin{aligned}
& \frac{\partial \boldsymbol{B}}{\partial t}=\frac{\partial \boldsymbol{B}^{*}}{\partial \tau}+\frac{\partial \boldsymbol{\xi}}{\partial t} \cdot\left(\nabla_{\boldsymbol{\xi}} \boldsymbol{B}^{*}\right)=\frac{\partial \boldsymbol{B}^{*}}{\partial \tau}-\boldsymbol{a} \cdot\left(\nabla_{\boldsymbol{\xi}} \boldsymbol{B}^{*}\right) \\
& \nabla_{\boldsymbol{x}} \boldsymbol{B}=\nabla_{\boldsymbol{\xi}} \boldsymbol{B}^{*}, \quad \nabla_{\boldsymbol{x}} \boldsymbol{w}=\nabla_{\boldsymbol{\xi}} \boldsymbol{w}^{*}+\nabla_{\boldsymbol{\xi}} \boldsymbol{a}=\nabla_{\boldsymbol{\xi}} \boldsymbol{w}^{*}
\end{aligned}
$$

hence, applying the above transformations to the terms on the left-hand side of evolution equation (3.10) yields to

$$
\begin{aligned}
& \frac{\partial \boldsymbol{B}^{*}}{\partial \tau}-\boldsymbol{a} \cdot\left(\nabla_{\boldsymbol{\xi}} \boldsymbol{B}^{*}\right)+\boldsymbol{w}^{*} \cdot\left(\nabla_{\boldsymbol{\xi}} \boldsymbol{B}^{*}\right)+\boldsymbol{a} \cdot\left(\nabla_{\boldsymbol{\xi}} \boldsymbol{B}^{*}\right)-\boldsymbol{B}^{*} \cdot\left(\nabla_{\boldsymbol{\xi}} \boldsymbol{w}^{*}\right) \\
= & \frac{\partial \boldsymbol{B}^{*}}{\partial \tau}+\boldsymbol{w}^{*} \cdot\left(\nabla_{\boldsymbol{\xi}} \boldsymbol{B}^{*}\right)-\boldsymbol{B}^{*} \cdot\left(\nabla_{\boldsymbol{\xi}} \boldsymbol{w}^{*}\right) .
\end{aligned}
$$

The similar transformations are easily applied to the terms in the parentheses on the right-hand side of evolution equation (3.10) as follows

$$
\begin{aligned}
& \boldsymbol{B}^{*} \cdot \frac{\partial \boldsymbol{B}^{*}}{\partial \tau}-\boldsymbol{a} \cdot\left(\nabla_{\boldsymbol{\xi}} \boldsymbol{B}^{*}\right) \cdot \boldsymbol{B}^{*} \\
+ & \boldsymbol{w}^{*} \cdot\left(\nabla_{\boldsymbol{\xi}} \boldsymbol{B}^{*}\right) \cdot \boldsymbol{B}^{*}+\boldsymbol{a} \cdot\left(\nabla_{\boldsymbol{\xi}} \boldsymbol{B}^{*}\right) \cdot \boldsymbol{B}^{*}-\boldsymbol{B}^{*} \cdot\left(\nabla_{\boldsymbol{\xi}} \boldsymbol{w}^{*}\right) \cdot \boldsymbol{B}^{*} \\
= & \boldsymbol{B}^{*} \cdot \frac{\partial \boldsymbol{B}^{*}}{\partial \tau}+\boldsymbol{w}^{*} \cdot\left(\nabla_{\boldsymbol{\xi}} \boldsymbol{B}^{*}\right) \cdot \boldsymbol{B}^{*}-\boldsymbol{B}^{*} \cdot\left(\nabla_{\boldsymbol{\xi}} \boldsymbol{w}^{*}\right) \cdot \boldsymbol{B}^{*} .
\end{aligned}
$$

Gathering all the transformed terms we obtain the following evolution equation for the magnetic induction in frame of reference $(\tau, \boldsymbol{\xi})$

$$
\begin{aligned}
& \boldsymbol{B}_{\tau}^{*}+\boldsymbol{w}^{*} \cdot \nabla_{\boldsymbol{\xi}} \boldsymbol{B}^{*}-\boldsymbol{B}^{*} \cdot \nabla_{\boldsymbol{\xi}} \boldsymbol{w}^{*} \\
= & \frac{1}{\left|\boldsymbol{B}^{*}\right|^{2}}\left(\frac{1}{2} \frac{\partial\left|\boldsymbol{B}^{*}\right|^{2}}{\partial \tau}+\boldsymbol{w}^{*} \cdot\left(\nabla_{\boldsymbol{\xi}} \boldsymbol{B}^{*}\right) \cdot \boldsymbol{B}^{*}-\boldsymbol{B}^{*} \cdot\left(\nabla_{\boldsymbol{\xi}} \boldsymbol{w}^{*}\right) \cdot \boldsymbol{B}^{*}\right) \boldsymbol{B}^{*} .
\end{aligned}
$$

The resulted equation is seen to be the same as the evolution equation in frame of reference $(t, \boldsymbol{x})$. This completes the proof.

\section{The incompleteness of the formulation}

Function $\lambda$ in (3.10) looks if it were obtained directly from (1.2) by the dot product of the former and the local vector $\boldsymbol{B}(t, \boldsymbol{x})$ as follows

$$
\boldsymbol{B} \cdot \boldsymbol{B}_{t}+\boldsymbol{w} \cdot(\nabla \boldsymbol{B}) \cdot \boldsymbol{B}-\boldsymbol{B} \cdot(\nabla \boldsymbol{w}) \cdot \boldsymbol{B}=\lambda \boldsymbol{B} \cdot \boldsymbol{B}
$$

nevertheless evolution equation (3.10) is obtainable by direct calculation of mixed derivative (3.9) in commutation condition (3.8). This is an evidence that evolution equation (3.10) is incomplete. Actually, the dot product of (3.10) and the local vector $\boldsymbol{B}(t, \boldsymbol{x})$ produces the trivial identity $\mathbf{0}=\mathbf{0}$, i. e., the evolution equation being under consideration 'works' only in planes normal to the local vectors $\boldsymbol{B}(t, \boldsymbol{x})$. The situation is clarified by the following 
Proposition 5.1. Evolution equation (3.10) for the magnetic induction is incomplete, i. e., it actually includes only two evolution equations for two scalar quantities.

Proof. Let domains $\mathcal{D}(t), t \geqslant t^{\prime}$, be parametrized using Cartesian orthogonal coordinates $\boldsymbol{x}=\left(x_{1}, x_{2}, x_{3}\right)$, hence, $\boldsymbol{B}=\left(B_{1}, B_{2}, B_{3}\right), \boldsymbol{w}=\left(w_{1}, w_{2}, w_{3}\right)$, and evolution equation (3.10) be rewritten in matrix form as the following quasilinear system of the first order

$$
\mathrm{A}_{0}(\mathbf{U}) \frac{\partial \mathbf{U}}{\partial t}+\sum_{\kappa=1}^{3} \mathrm{~A}_{\kappa}(\mathbf{U} ; \boldsymbol{w}) \frac{\partial \mathbf{U}}{\partial x_{\kappa}}=\mathbf{G}(\mathbf{U} ; \nabla \boldsymbol{w})
$$

where

$$
\mathbf{U}=\left(\begin{array}{c}
B_{1} \\
B_{2} \\
B_{3}
\end{array}\right), \quad \mathrm{A}_{0}(\mathbf{U})=\left(\begin{array}{rrr}
|\boldsymbol{B}|^{2}-B_{1} B_{1} & -B_{1} B_{2} & -B_{1} B_{3} \\
-B_{2} B_{1} & |\boldsymbol{B}|^{2}-B_{2} B_{2} & -B_{2} B_{3} \\
-B_{3} B_{1} & -B_{3} B_{2} & |\boldsymbol{B}|^{2}-B_{3} B_{3}
\end{array}\right)
$$

$\mathrm{A}_{\kappa}(\mathbf{U} ; \boldsymbol{w})=\mathrm{A}_{0}(\mathbf{U}) \mathrm{C}_{\kappa}(\boldsymbol{w})=\mathrm{C}_{\kappa}(\boldsymbol{w}) \mathrm{A}_{0}(\mathbf{U}), \quad \mathrm{C}_{\kappa}(\boldsymbol{w})=w_{\kappa} \operatorname{diag}(1,1,1)=w_{\kappa} \mathrm{E}$,

$$
\mathbf{G}(\mathbf{U} ; \nabla \boldsymbol{w})=\left(\begin{array}{c}
|\boldsymbol{B}|^{2} \phi_{1}(\boldsymbol{B} ; \nabla \boldsymbol{w})-\phi(\boldsymbol{B} ; \nabla \boldsymbol{w}) B_{1} \\
|\boldsymbol{B}|^{2} \phi_{2}(\boldsymbol{B} ; \nabla \boldsymbol{w})-\phi(\boldsymbol{B} ; \nabla \boldsymbol{w}) B_{2} \\
|\boldsymbol{B}|^{2} \phi_{3}(\boldsymbol{B} ; \nabla \boldsymbol{w})-\phi(\boldsymbol{B} ; \nabla \boldsymbol{w}) B_{3}
\end{array}\right)
$$

$\phi_{1}, \phi_{2}$, and $\phi_{3}$ being linear forms and $\phi$ being a quadratic form in the components of the $\boldsymbol{B}$-field as follows

$$
\phi(\boldsymbol{B} ; \nabla \boldsymbol{w})=\sum_{\kappa=1}^{3} \sum_{\iota=1}^{3} B_{\kappa} B_{\iota} \frac{\partial w_{\kappa}}{\partial x_{\iota}}=\sum_{\iota=1}^{3} B_{\iota}\left(\sum_{\kappa=1}^{3} B_{\kappa} \frac{\partial w_{\kappa}}{\partial x_{\iota}}\right)=\sum_{\iota=1}^{3} B_{\iota} \phi_{\iota}(\boldsymbol{B} ; \nabla \boldsymbol{w}) .
$$

We use matrix notation $\mathbf{U}$ for the dependent variables in matrix formulation (5.2) of evolution equation (3.10) and its constitutive parts and vector notation $\boldsymbol{B}$ in the scalar functions and the entries of the matrices.

Matrix $A_{0}$ is singular: $\operatorname{det} A_{0}=0, \operatorname{rank} A_{0}=2$, but being real symmetric it has real eigenvalues: $\lambda_{1}=0, \lambda_{2}=|\boldsymbol{B}|^{2}, \lambda_{3}=|\boldsymbol{B}|^{2}$, and complete sets of the left (rows) and the right (columns) normalized real eigenvectors

$$
\mathrm{L}(\mathbf{U})=\mathrm{R}^{-1}(\mathbf{U})=|\boldsymbol{B}|^{-2}\left(\begin{array}{rrr}
B_{1} B_{3} & B_{2} B_{3} & B_{3} B_{3} \\
-B_{1} B_{3} & -B_{2} B_{3} & B_{1} B_{1}+B_{2} B_{2} \\
-B_{2} B_{1} & B_{1} B_{1}+B_{3} B_{3} & -B_{2} B_{3}
\end{array}\right),
$$




$$
\mathrm{R}(\mathbf{U})=\mathrm{L}^{-1}(\mathbf{U})=\left(\begin{array}{ccc}
\frac{B_{1}}{B_{3}} & -\frac{B_{3}}{B_{1}} & -\frac{B_{2}}{B_{1}} \\
\frac{B_{2}}{B_{3}} & 0 & 1 \\
1 & 1 & 0
\end{array}\right)
$$

The left and the right eigenvectors are normalized in such a way to diagonalize matrix $A_{0}$ as follows

$\mathrm{L}(\mathbf{U}) \mathrm{A}_{0}(\mathbf{U}) \mathrm{R}(\mathbf{U})=\operatorname{diag}\left(0,|\boldsymbol{B}|^{2},|\boldsymbol{B}|^{2}\right) \equiv \mathrm{Q}(\mathbf{U}), \quad \mathrm{A}_{0}(\mathbf{U})=\mathrm{R}(\mathbf{U}) \mathrm{Q}(\mathbf{U}) \mathrm{L}(\mathbf{U})$

Applying the above property to system (5.2) yields to

$$
\mathrm{Q}(\mathbf{U})\left(\mathrm{L}(\mathbf{U}) \frac{\partial \mathbf{U}}{\partial t}+\sum_{\kappa=1}^{3} \mathrm{C}_{\kappa}(\boldsymbol{w}) \mathrm{L}(\mathbf{U}) \frac{\partial \mathbf{U}}{\partial x_{\kappa}}\right)=\mathbf{H}(\mathbf{U} ; \nabla \boldsymbol{w}),
$$

where the source term on the right-hand side has the following explicit form

$$
\mathbf{H}(\mathbf{U} ; \nabla \boldsymbol{w}) \equiv \mathrm{L}(\mathbf{U}) \mathbf{G}(\mathbf{U} ; \nabla \boldsymbol{w})=\left(\begin{array}{c}
0 \\
|\boldsymbol{B}|^{2} \phi_{3}(\boldsymbol{B} ; \nabla \boldsymbol{w})-B_{3} \phi(\boldsymbol{B} ; \nabla \boldsymbol{w}) \\
|\boldsymbol{B}|^{2} \phi_{2}(\boldsymbol{B} ; \nabla \boldsymbol{w})-B_{2} \phi(\boldsymbol{B} ; \nabla \boldsymbol{w})
\end{array}\right)
$$

whereas the terms on the left-hand side need to be simplified using a proper transformation of the dependent variables $\mathbf{U}$.

The proper choice of the variable transformation is prompted by the differential terms on the the left-hand side of system (5.3) leading to the following matrixcolumn product

$$
|\boldsymbol{B}|^{2} \mathrm{~L}(\mathbf{U}) \mathrm{d} \mathbf{U}=\left(\begin{array}{ccc}
B_{1} B_{3} \mathrm{~d} B_{1}+ & B_{2} B_{3} \mathrm{~d} B_{2}+ & B_{3} B_{3} \mathrm{~d} B_{3} \\
-B_{1} B_{3} \mathrm{~d} B_{1}- & B_{2} B_{3} \mathrm{~d} B_{2}+\left(B_{1} B_{1}+B_{2} B_{2}\right) \mathrm{d} B_{3} \\
-B_{2} B_{1} \mathrm{~d} B_{1}+\left(B_{1} B_{1}+B_{3} B_{3}\right) \mathrm{d} B_{2}- & B_{2} B_{3} \mathrm{~d} B_{3}
\end{array}\right),
$$

where scalar multiplier $|\boldsymbol{B}|^{2}$ represents the non-zero entries of matrix $\mathrm{Q}(\mathbf{U})$.

The 1-forms given by the entries of the resulted column vector above and denoted below respectively as $\omega_{1}, \omega_{2}$, and $\omega_{3}$, are evidently to be integrable, the integrating factors being as follows

$$
\theta_{1}(\boldsymbol{B})=\frac{1}{B_{3}\left(B_{1}^{2}+B_{2}^{2}+B_{3}^{2}\right)}, \theta_{2}(\boldsymbol{B})=\frac{1}{B_{2}^{2} \sqrt{B_{1}^{2}+B_{3}^{2}}}, \theta_{3}(\boldsymbol{B})=\frac{1}{B_{3}^{2} \sqrt{B_{1}^{2}+B_{2}^{2}}}
$$


Hence, we find that

$$
\left\{\begin{array}{l}
\theta_{1}(\boldsymbol{B}) \omega_{1}=\mathrm{d} v_{1}=\mathrm{d} \sqrt{B_{1}^{2}+B_{2}^{2}+B_{3}^{2}}, \\
\theta_{2}(\boldsymbol{B}) \omega_{2}=\mathrm{d} v_{2}=\mathrm{d} \frac{\sqrt{B_{1}^{2}+B_{3}^{2}}}{B_{2}}, \\
\theta_{3}(\boldsymbol{B}) \omega_{3}=\mathrm{d} v_{3}=\mathrm{d} \frac{\sqrt{B_{1}^{2}+B_{2}^{2}}}{B_{3}},
\end{array},\right.
$$

and the new dependent variables are $\mathbf{V}(\mathbf{U})=\left(v_{1}, v_{2}, v_{3}\right)$.

Eventually, the first differential equation of system (5.3) vanishes, whereas the remaining two ones read

$$
\frac{\partial}{\partial t}\left(\begin{array}{l}
v_{2} \\
v_{3}
\end{array}\right)+\sum_{\kappa=1}^{3}\left(\begin{array}{cc}
w_{\kappa} & 0 \\
0 & w_{\kappa}
\end{array}\right) \frac{\partial}{\partial x_{\kappa}}\left(\begin{array}{l}
v_{2} \\
v_{3}
\end{array}\right)=\left(\begin{array}{l}
p_{2}\left(v_{1}, v_{2}, v_{3} ; \nabla \boldsymbol{w}\right) \\
p_{3}\left(v_{1}, v_{2}, v_{3} ; \nabla \boldsymbol{w}\right)
\end{array}\right),
$$

where

$$
\left\{\begin{array}{l}
p_{2}\left(v_{1}, v_{2}, v_{3} ; \nabla \boldsymbol{w}\right)=\left[\theta_{2}(\boldsymbol{B}) h_{2}(\boldsymbol{B} ; \nabla \boldsymbol{w})\right]_{\mathbf{U} \rightarrow \mathbf{V}}, \\
p_{3}\left(v_{1}, v_{2}, v_{3} ; \nabla \boldsymbol{w}\right)=\left[\theta_{3}(\boldsymbol{B}) h_{3}(\boldsymbol{B} ; \nabla \boldsymbol{w})\right]_{\mathbf{U} \rightarrow \mathbf{V}},
\end{array}\right.
$$

and $h_{2}, h_{3}$ are the two last entries of column vector $\mathbf{H}$ (5.4). Both equations of system (5.6) are coupled through source terms $p_{2}$ and $p_{3}$.

Finding the above system completes the proof.

\section{Conclusions of the formulation}

1. Evolution equation (1.2) is uniquely determined by diffeomorphisms $\boldsymbol{\varphi}_{\boldsymbol{w}}$, $\varphi_{B}$ and $\bar{\varphi}_{B}$. This means that function $\lambda$ explicitly depends on the local values of fields $\boldsymbol{B}(t, \boldsymbol{x})$ and $\boldsymbol{w}(t, \boldsymbol{x})$ and the partial derivatives of $\boldsymbol{B}(t, \boldsymbol{x})$ as it is seen from formulation (3.10) of the evolution equation, rather than being 'a free function'.

2. Evolution equation (1.2) is influenced by velocity field $\boldsymbol{w}(t, \boldsymbol{x})$, nevertheless substituting velocity field $\boldsymbol{u}(t, \boldsymbol{x})$ into (1.2) does not convert the former into magnetic induction equation (1.3), since in both formulations (1.1) and (1.3) of the magnetic induction equation and in evolution equation (1.2) for the magnetic induction the velocity fields have quite different meanings.

3. Evolution equation (1.2) is Galilean invariant similarly to formulation (1.3) of the magnetic induction equation.

4. Evolution equation (1.2) is incomplete, since it is reduced to system (5.6) of two partial differential equations for dependent variables $v_{2}, v_{3}(5.5)$. System (5.6) needs to be supplemented with a constraint imposed on variables $\left(v_{1}, v_{2}, v_{3}\right)$, either algebraic or differential, to admit the well-posed formulations $[4,8]$ of IBVP. 
5. Variable $v_{1}(5.5)$ is introduced similarly to variables $v_{2}, v_{3}$ using the left eigenvectors but variable $v_{1}$ turns out to be blind to the sign of component $B_{1}$ of the $\boldsymbol{B}$-field, contrary to variables $v_{2}, v_{3}$ accounting for the signs of components $B_{2}$ and $B_{3}$. Therefore, an other choice for $v_{1}$ may be more appropriate.

\section{References}

1. R. Abraham, J. E. Marsden, T. Ratiu, Manifolds, Tensor Analysis, and Applications, Springer, NY, 1988.

2. J. Birn, E. Priest (Eds.), Reconnection of Magnetic Fields, Cambridge University Press, Cambridge, 2007.

3. D. Biskamp, Magnetic Reconnection in Plasmas, Cambridge University Press, Cambridge, 2000.

4. R. Courant, D. Hilbert, Methods of Mathematical Physics, Vol. II, Partial Differential Equations, John Wiley \& Sons, Inc., NY, 1962.

5. P. A. Davidson, An Introduction to Magnetohydrodynamics, Cambridge University Press, Cambridge, 2001.

6. S.K. Godunov, Symmetric form of the equations of magnetohydrodynamics, Numer. Methods Mech. Cont. Media, 3 (1972), $26-34$.

7. G. Hornig, K. Schindler, Magnetic topology and the problem of its invariant definition, Phys. Plasmas, 3 (1996), 781-791.

8. F. John, Partial Differential Equations, Springer, NY, 1982.

9. W. A. Newcomb, Motion of magnetic lines of force, Ann. Phys, 3 (1958), 347-385.

10. W. Pauli, Theory of Relativity, Pergamon Press, London, 1958.

11. E. Priest, T. Forbes, Magnetic Reconnection: MHD Theory and Applications, Cambridge University Press, Cambridge, 2000.

12. D. P. Stern, The motion of magnetic field lines, Rep. Progr. Phys., 6 (1966), $147-173$.

13. K. ZoraWski, Über die Erhaltung der Wirbelbewegung, C. R. Acad. Sci. Cracovie, $1900,335-341$. 